\title{
LETTERS
}

\section{Taking a long-term view of body sculpting}

The CMAJ Practice article by ProsperiPorta and colleagues outlined short- and intermediate-term toxicities of some body sculpting products. ${ }^{1}$ Others have reported long-term histopathological reactions to injections of foreign substances. ${ }^{2}$ Metabolic effects have also been reported, adding to the overall picture of the consequences of body sculpting products. ${ }^{3}$

One less frequently studied but potentially serious consequence of body sculpting injections is their longer-term effect on immune dysregulation, and thereafter autoimmunity, that can potentially arise with chronic exposure to foreign products. ${ }^{4,5}$ An autoimmune/inflammatory syndrome has been linked to foreign materials that may create adjuvancy. ${ }^{4}$ Some of the clinical presentations may include atypical multiple sclerosis, arthritides, possible Sjogren syndrome and antiphospholipid syndromes, among others. ${ }^{4}$ Silicone products are infamous in this regard; some silicone implants have been associated with atypical lymphomas. ${ }^{4}$ Variable dysautonomia and peripheral neuropathy can further add to the already complicated scenario. ${ }^{5}$ The presentations of such immune dysregulation disorders are often insidious and confuse both patient and physician. Multiple body systems may be involved, and patients often see many doctors in search of a diagnosis and effective treatment. ${ }^{4}$

Mast cell activation may be linked to body sculpting products and create an allergic profile that, at times, may mimic mastocytosis. Mast cell activation is usually polyclonal, but in its chronic form, may be associated with autoimmunity. Indeed, some have wondered whether immune dysregulation disorders are triggered by mast cell hyperreactivity. ${ }^{4}$ In particular, oils may act as adjuvants, as exemplified by incomplete Freund's adjuvant. ${ }^{4}$ Many pathology studies of such local reactions do not specifically detail the presence or frequency of mast cells in situ. ${ }^{4}$

Some in our society seem obsessed with attaining particular body image stereotypes. The frequency with which foreign materials are injected to accomplish this goal is not only considerable, but the array of products to do so has increased to match the high demand. Should long-term exposure to foreign materials lead to chronic immune stimulation and its adverse consequences, we can expect an epidemic of autoimmune or mast cell-guided pathologies. The presentation of these diseases will continue to be a major issue, given the long-term nature of disease evolution, and product removal will become increasingly important. Removal of solid foreign bodies can at times lead to complete or partial resolution of symptoms. When the product is liquid-based (e.g., oils or emulsions), removal is much more challenging; there is potential for these products to spread far beyond the initial injection site. Anticipating these potential complications is important because the multiple complex presentations with adjuvancy from foreign products will not often be recognized at first glance.

\section{Nevio Cimolai MD}

Physician, Faculty of Medicine, The University of British Columbia, Vancouver, BC

Cite as: CMAJ 2020 December 7;192: E1730. doi: 10.1503/cmaj.77136

\section{References}

1. Prosperi-Porta G, Oleynick C, Vaughan S. Myositis from intramuscular oil injections in a bodybuilder. CMAJ 2020;192:E480

2. AbdullGaffar B. Illicit injections in bodybuilders: a clinicopathological study of 11 cases in 9 patients with a spectrum of histological reaction patterns. Int J Surg Pathol 2014;22:688-94.

3. Eldrup E, Theilade S, Lorenzen M, et al. Hypercalcemia after cosmetic oil injections: unraveling etiology, pathogenesis, and severity. J Bone Miner Res 2020 Sept. 15 [Epub ahead of print]. doi: 10.1002/jbmr.4179.

4. Cimolai N. Mast cell biology and linkages for non-clonal mast cell activation syndrome and autoimmune/inflammatory syndrome induced by adjuvants. SN Comp Clin Med 2020 Oct. 8 [Epub ahead of print]. doi: 10.1007/s423399-020-00494-8.

5. Cimolai N. Mast cell biology in the context of dysautonomia and neuropathy. Clin Immunol 2020; 215:108417.

Competing interests: None declared. 